\title{
Online Learning for Spectrum Sensing and Reconfigurable Antenna Control
}

\author{
Kevin Wanuga, Student Member, IEEE, Nikhil Gulati, Student Member, IEEE, Harri Saarnisaari, Member, IEEE \\ and Kapil R. Dandekar, Senior Member, IEEE
}

\begin{abstract}
Efficient dynamic spectrum access (DSA) policies rely on accurate spectrum sensing information to exploit spectrum white space optimally. Obtaining accurate channel state information (CSI) from local spectrum measurements is made difficult by wireless signal fading and the presence of thermal noise which distorts measured signals and leads to uncertainty regarding the occupancy of spectrum resources. Electrically reconfigurable antenna systems (ERAS) offer the system designer an additional degree of freedom to exploit pattern and polarization diversity to improve the accuracy of local spectrum sensing decisions. We propose a learning technique to exploit pattern and polarization diversity offered by ERAS to improve spectrum sensing accuracy. While the proposed approach is designed to work within the unique design constraints of reconfigurable antennas, the approach is not antenna specific and will work with a wide variety of reconfigurable antenna designs. Validation of system performance is provided from measurements taken using the wireless open access research platform (WARP) software defined radio (SDR) platform in an indoor office environment.
\end{abstract}

\section{INTRODUCTION}

Research in spectrum sensing for dynamic spectrum access (DSA) applications has been broad and varied e.g., [1]. Different techniques have been proposed to limit the occurrence of spectrum sensing errors that can result from wireless signal fading and thermal noise which distort measured signals and leads to uncertainty about the state of the wireless spectrum resource. Since wireless signal fading is environment specific, the performance of spectrum sensors is link dependent. This link dependence can result in sub-optimal performance when sensors are located in the presence of a deep fade or shadowed.

To address this problem, it has previously been proposed to use antenna array techniques like synthetic apertures and diversity algorithms to improve spectrum sensing reliability [2]-[8]. These techniques are capable of improving system performance and reliability by exploiting the spatial diversity available to multi-antenna arrays.

electrically reconfigurable antenna systems (ERAS) are an emerging class of antenna systems that are capable of altering antenna pattern and polarization through the use of a control network of electric switches (e.g., solid state switches, or micro-electromechanical systems (MEMS)), or tunable resonant elements (e.g., varactors) embedded in the antenna structure [9]-[12]. These control networks allow for rapid reconfiguration of antennas making it possible to adapt to changing environmental conditions on a transmission by transmission basis. Reconfigurable antennas offer the potential to extend the capabilities of both single antenna systems and
Unlike conventional diversity techniques, the challenge of reconfigurable antenna configuration control is uniquely constrained. ERAS can exist in only one antenna configuration at any given time, making it impossible to have complete channel state information (CSI) over all antennas states at any given time. As a result, ERAS configuration control algorithms must balance the benefit of exploring the antenna state space with the cost of using the system in suboptimal antenna configurations. This constraint has been identified in previous applications of ERAS for point-to-point multipleinput-multiple-output (MIMO) wireless links [13]-[15].

The proposed control algorithm provides a method for the optimized selection of ERAS states for the purposes of minimizing spectrum sensing errors. This algorithm balances the trade-off between exploration of the performance of each ERAS configuration, and the exploitation of the ERAS configuration which is best suited for meeting the design objective. Experimental validation of the proposed technique was carried out using empirical wireless channel data from an indoor office environment.

\section{SySTEM Model}

Typical spectrum sensing decisions are made from local measurement of the wireless spectrum resource that the opportunistic user is hoping to exploit. An overview of a typical spectrum sensing architecture can be seen in Figure 1. After the signal has been captured and sampled, a transform on the signal is done to produce a statistic, $r[n]$, which is used to make a decision, $\hat{h}$, on the state of the wireless spectrum, ( 0 = vacant, 1 = occupied). This decision is made by partitioning the domain of $r[n]$ into regions that are most likely to represent that the channel is either occupied or vacant. These decision regions are dictated by the distribution of the test statistic under either the null hypothesis (vacant) or the alternative hypothesis (occupied). Since the test statistic is derived from a transformation of the measured spectrum, the corresponding distribution is determined by environment specific fading and subject to change based on variation in the propagation environment.

To mitigate the variability in performance that comes from the changing wireless environment, a modification of the existing sensor structure is proposed that incorporates the use of a reconfigurable antenna and the algorithm which is capable of configuring the various antenna states. An overview of this system can be seen in Figure 2. This two stage algorithm first estimates the performance of the spectrum sensor using 


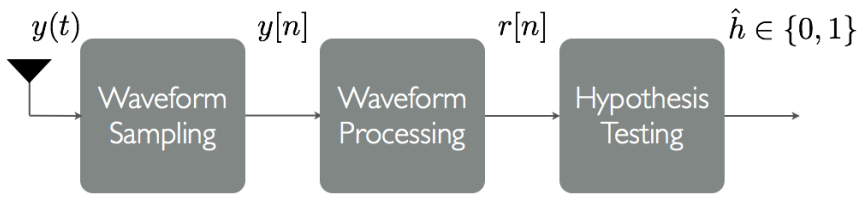

Fig. 1: System diagram of conventional spectrum sensor.

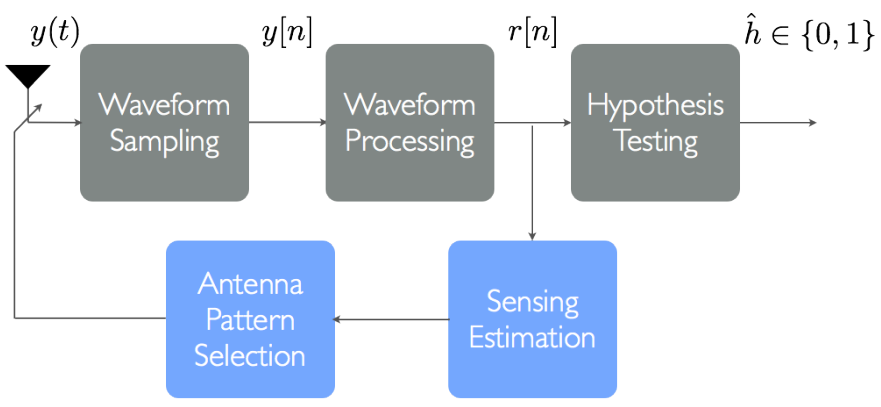

Fig. 2: System diagram of spectrum sensor incorporating ERAS.

the given antenna configuration. This estimate is obtained through characterization of the test statistic, $r[n]$. The estimate of sensor performance is then used to compute a weighted reward which indicates the utility of using the current state for spectrum sensing which is used to select the appropriate antenna state for the next sensing phase. We provide more details in Sec II-B

\section{A. Sensing Performance Estimation}

Assuming that the spectrum sensor is an energy detector, the distribution of the test statistic, $r[n]$, is determined by the distribution of the noise energy under the null hypothesis, and the energy of the signal and noise present under the alternative hypothesis. There have been a wide variety of statistical models used to represent the energy of signals with wireless fading, and while noise energy is typically assumed to be additive white Guassian noise (AWGN), it is desirable to be able to account for non-Guassian sources of noise as well. For this reason, we model the test statistic as a mixture of Gamma random variables. Gamma random variables are a useful model since i) they generalize a variety of common fading models in the exponential family such as Rayleigh, Nakagami, and $\chi^{2}$, and ii) they can approximate other models such as lognormal random variables with reasonable accuracy [16], [17]. A mixture model is used to represent the fact that the statistic is distributed differently depending on the true state of the channel, and this true state is obscured by the non-zero probability of error in spectrum sensing decisions.

The expectation maximization (EM) algorithm provides a robust mechanism for estimating the parameter of various mixture components in a mixture model despite this uncertainty about the state of the channel at the time the energy samples are collected [18]. Additionally, the EM algorithm has the added benefit of converging efficiently when the mixture components have distributions in the exponential family [19]. This procedure iteratively converges on maximum likelihood estimates (MLE) for the given parameters by:

1) Selecting initial parameter estimates

2) Computing a conditional likelihood based on initial parameter estimates

3) Obtaining revised parameter estimates as the parameter values that maximize the computed conditional likelihood function

4) Repeating steps 2) and 3) until convergence

\section{B. Antenna Configuration Selection Technique}

Once the estimates are obtained for the parameters of both the noise and signal distributions, a reward function, $\mu_{i}$ is computed to establish how well a given antenna configuration, $i$, is optimized for spectrum sensing. There are two types of error that can occur from this hypothesis test. A false positive occurs when the spectrum sensor decides the wireless spectrum is occupied when it is in fact vacant. A false negative occurs when the spectrum sensor decides the wireless spectrum is vacant when it is in fact occupied. Since a test can be devised that produces arbitrarily small probability of false positive or false negative at the cost of increasing the other, a more effective method is minimizing the probability of one type of error under a constraint on the other. More specifically, the reward for a given configuration will be minimizing the probability of false negative given a constraint on the probability of false positive, or selecting the antenna configuration which provides the optimal constant false alarm rate (CFAR) detector [20].

If an energy detector is being used, then the image of the test statistic, $r[n]$ is the non-negative real line. Since the likelihood of a signal being present increases with an increase in the value of $r[n]$, the decision rule is straightforward. If the $r[n] \geqslant \gamma$ then the detector decides that the spectrum is occupied. The critical value, $\gamma$, is then determined to meet the false positive constraint such that

$$
P_{+}=\int_{0}^{\gamma} \frac{1}{\Gamma\left(\alpha_{0}\right)} x^{\alpha_{0}-1} e^{-\beta_{0} x} d x
$$

where, $\alpha_{0}, \beta_{0}$ are the parameters of the noise power estimated from the EM algorithm. This quantity represents the probability that the vacant spectrum noise power exceeds the critical value, $\gamma$. The reward, $\mu$, can then be calculated as

$$
\begin{aligned}
\mu & =1-P_{-} \\
& =\int_{\gamma}^{\infty} \frac{1}{\Gamma\left(\alpha_{1}\right)} x^{\alpha_{1}-1} e^{-\beta_{1} x} d x,
\end{aligned}
$$

where, $\alpha_{1}, \beta_{1}$ are the statistic, $r[n]$, from the alternative hypothesis, computed from the EM algorithm, and $\gamma$ is determined from (1). The reward, $\mu_{i}$ is calculated based on different estimates for each test statistic using each antenna configuration.

In this way, the reward $0<\mu_{i}<1$ is a calculation of the probability of correctly identifying an occupied channel 
given a constraint on the rate of false positives. We define the optimal reward, $\mu^{*}=\max _{i} \mu_{i}$, as the highest probability of correct detection achievable from any of the antenna configurations The strategy for selecting an antenna configuration is designed to balance the benefit of learning this reward for each configuration with the cost of using antenna configurations that have sub-optimal reward. The strategy for balancing this exploration and exploitation is based on a variant of a solution to the multi-armed bandit problem [21]. We define the regret, $R(n)$, of an antenna configuration selection strategy as

$$
R(n)=\mu^{*} n-\mu_{i} \sum_{i=1}^{K} \mathbf{E}\left[T_{i}(n)\right]
$$

where, $\mathbf{E}\left[T_{i}(n)\right]$ is the expected number of times antenna configuration $i$ is played after $n$ total plays. Regret identifies the loss in reward that comes from using sub-optimal antenna configurations. It has previously been shown that any antenna selection strategy whose reward, $\mu \rightarrow \mu^{*}$ will have regret, $R(n)=O(\ln (n))$. One such strategy for achieving optimal rate of regret growth is the upper confidence bound (UCB) algorithm derived in [21], which assigns each antenna configuration a priority

$$
V_{i}=\mu_{i}+\sqrt{\frac{2 \ln (n)}{n_{i}}},
$$

where $n$ is the total number of sensing decisions made, and $n_{i}$ is the number of times antenna configuration, $i$, has been used for spectrum sensing. The proposed strategy uses the antenna configuration which maximizes this priority, $V_{i}$, for the next spectrum sensing decision. This strategy includes both an estimate for the true reward, $\mu_{i}$, of using antenna configuration, $i$, as well as a weight that increases if configuration $i$ is being used infrequently.

\section{EXPERIMENT OVERVIEW}

To verify the performance of this approach, wireless measurement were taken in the Bossone Research Enterprise Center on the campus of Drexel University. The environment was selected to be representative of an indoor office environment. An overview of the physical link topology can be seen in Figure 3. In this experiment, a single transmitter broadcast bursty traffic on a WIFI channel located at $2.462 \mathrm{GHz}$, and one receiver used an energy detector to attempt to detect the occupancy of the channel.

In this experiment, traffic was synchronized with spectrum sensing decisions. At each time slot, the transmitter would flip a coin to determine whether to transmit or not. At that same time, the receiver would measure the wireless spectrum and make a decision on whether to transmit or not. Relevant parameters for the experiment can be seen in Table I.

The receiver was deployed with a reconfigurable Alford loop antenna (RALA) [22], a pattern reconfigurable antenna with 4 directional antenna patterns that range $360^{\circ}$ in azimuth and an omni-directional antenna pattern. The antenna pattern of each antenna configuration can be seen in Figure 4.

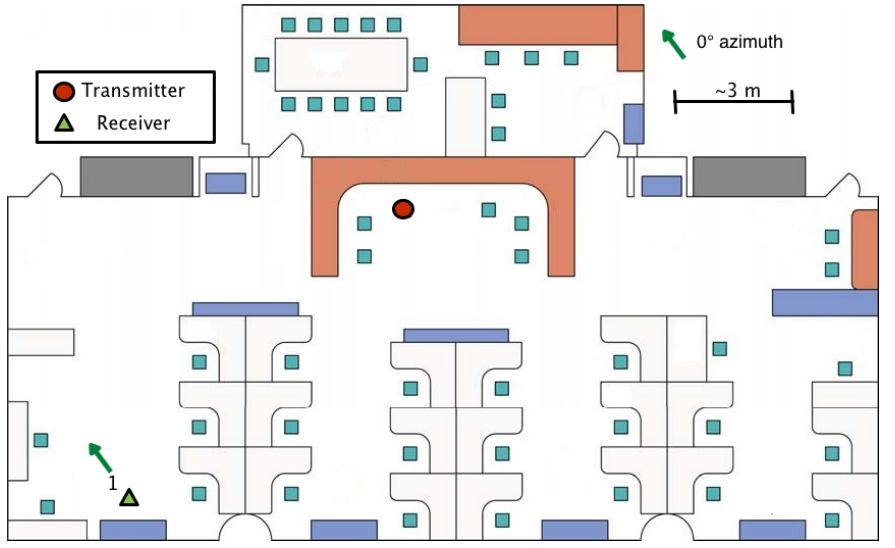

Fig. 3: Overview of radio locations for spectrum sensing experiment.

\begin{tabular}{l|c} 
Parameter & Values \\
\hline \hline Probability of vacancy, $\pi_{0}$ & 0.5 \\
Transmit power, $P_{\mathrm{Tx}}$ & $1 \mathrm{dBm}$ \\
Total samples, $N$ & 3000 \\
EM iterations, $T$ & 15
\end{tabular}

TABLE I: Table of relevant experiment parameters.

Each radio used is a 3rd generation version of the wireless open access research platform (WARP) software defined radio (SDR) platform developed by Rice University [23]. Coordination and control of the radios was managed over a hardwired ethernet interface. All data generation for the transmitters and data analysis for the receivers was performed in MATLAB.

\section{Measured Results}

The measured configuration reward, $P_{\mathrm{CD}}$, with false alarm rate constrained to $10 \%$ can be seen in Table II. As the results indicate, spectrum sensing performance for this radio is poor, most likely due to low transmit power of the broadcasting

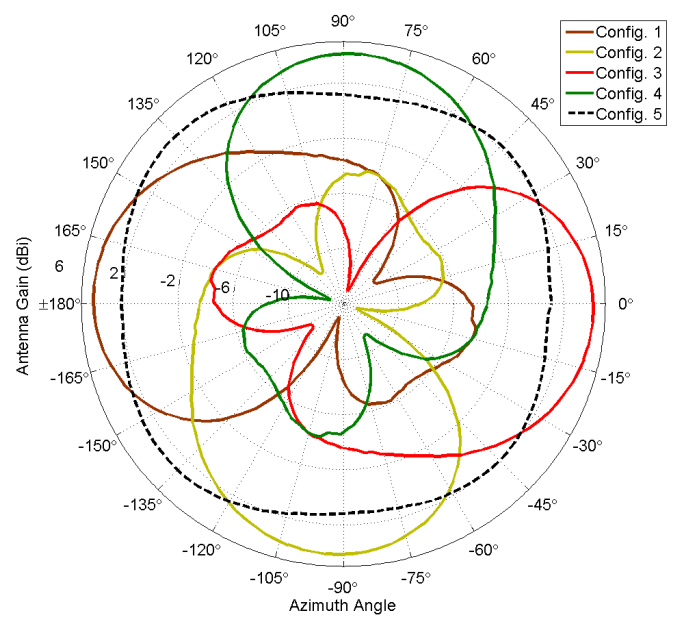

Fig. 4: Antenna patterns for each of the antenna configuration of the RALA. 


\begin{tabular}{c|c} 
Antenna Configuration & $P_{\mathrm{CD}}$ \\
\hline \hline 1 & .11 \\
2 & .41 \\
3 & .17 \\
4 & .12 \\
5 & .55
\end{tabular}

TABLE II: $P_{\mathrm{CD}}$ for each antenna configuration with constrained $P_{\mathrm{FA}}=0.1$.

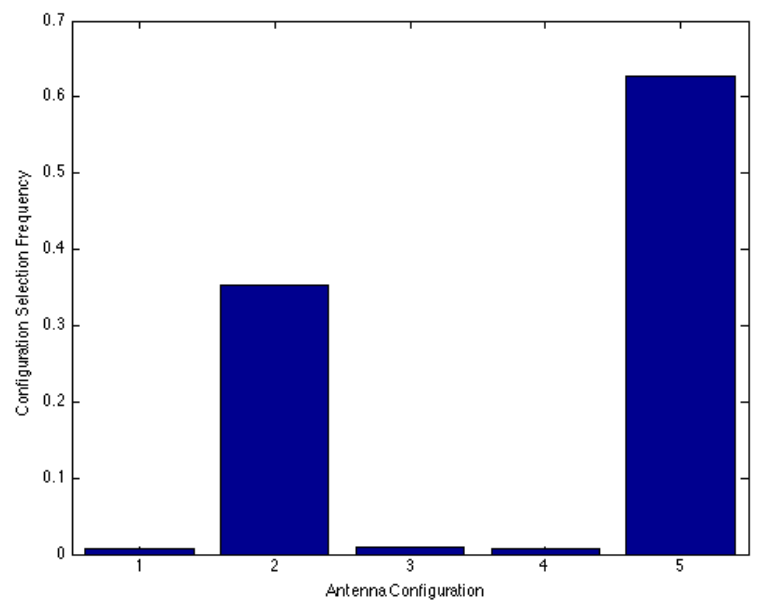

Fig. 5: Frequency of selection for each antenna configuration.

node. The omni-directional antenna configuration offers the best performance and with antenna configuration 2 offering better performance than any other directional pattern.

The UCB algorithm is designed to make use of antenna configurations proportional to their relative performance. This behavior is clear from observing the frequency of accessing each antenna configuration shown in Figure 5. Antenna configuration 5 (omni-directional) is selected for the large majority of spectrum sensing samples over the duration of the 3000 samples collected. Antenna configuration 2 is selected for approximately $30 \%$ of spectrum samples owing to its relatively strong performance. Antenna configurations with poor performance are largely unused. The results indicate a strong dependence on the environment in which the measurements are taken. It is reasonable to assume that an omni-directional antenna would offer the best performance in a rich scattering environment. In outdoor environments or in environments with sparse multi-path or at low signal-to-noise ratio (SNR), ERAS with directional patterns have demonstrated performance gains over conventional antennas [24], [25]. Therefore, we believe the sensing performance of directional modes of the antenna may out perform omni-directional mode in such cases. Further, in dense cognitive radio networks, it is possible to use omnidirectional antenna modes to maximize sensing accuracy and then use one of the directional modes for spectrum access to reduce interference and maximize network capacity.

Figure 6 shows the effective performance of the ERAS spectrum sensor after 3000 spectrum samples. The optimal ROC shows the performance of a controller with perfect CSI. The

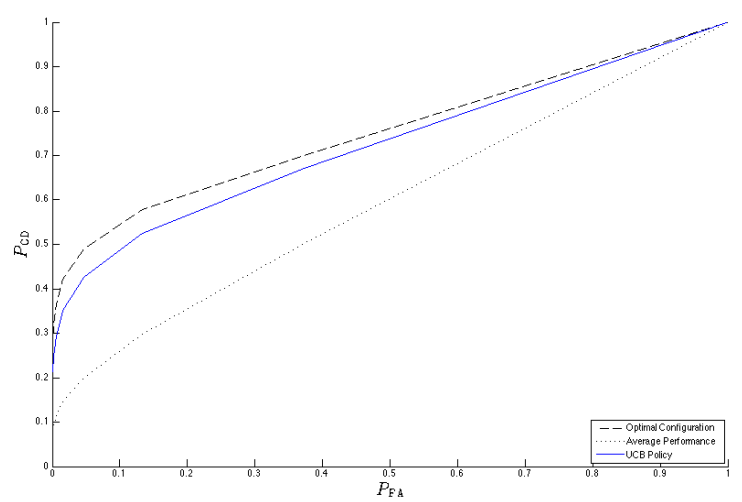

Fig. 6: Effective ROC of ERAS spectrum sensor.

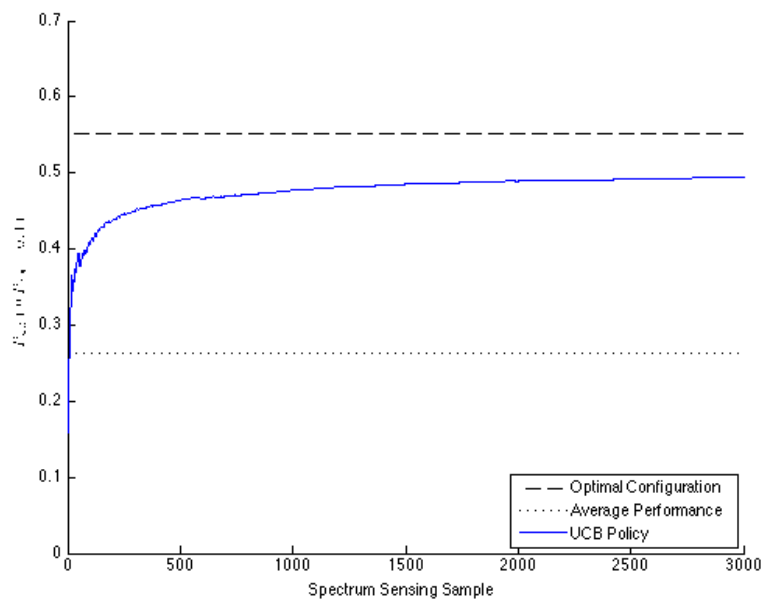

Fig. 7: Effective $P_{\mathrm{CD}}$ of ERAS spectrum sensor over time.

UCB approach performs to $89.7 \%$ of optimal performance, and $188 \%$ of average performance that would be seen with a random selection strategy.

Figure 7 shows the rate of convergence of $P_{\mathrm{CD}}\left(@ P_{\mathrm{FA}}=0.1\right)$ UCB algorithm in time. This convergence in performance is quite rapid, converging to $80 \%$ of optimal performance within 200 samples. As discussed previously, the UCB algorithm will converge asymptotically on the optimal performance as the number of captured samples tends towards infinity.

The experimental regret is shown in Figure 8. Visual inspection of the regret growth shows that it appears to be sublinear, however it is difficult to verify whether growth is truly logarithmic from experimental results. Achieving sub-linear regret will result in better performance than an exhaustive training or randomized approach that will have linear growth in regret. Further improvements in regret growth may be obtained from modification of the antenna control algorithm.

Table III shows the MLE of $\alpha$ and $\beta$ parameters of the signal distribution that can only be obtained with perfect information about the occupancy of the channel. Additionally, the EM estimates of the $\alpha$ and $\beta$ parameters obtained from the ERAS with state uncertainty are shown. As the results show, the 


\begin{tabular}{l|c|c} 
Antenna Configuration & $\alpha$ & $\beta$ \\
\hline \hline config. 1 & & \\
-MLE parameters & .97 & .0006 \\
-EM parameters & .94 & .0006 \\
\hline config. 2 & & \\
-MLE parameters & .66 & .002 \\
-EM parameters & .66 & .003 \\
\hline config. 3 & & \\
-MLE parameters & .93 & .0008 \\
-EM parameters & .86 & .0009 \\
\hline config. 4 & & \\
-MLE parameters & .96 & .0006 \\
-EM parameters & .84 & .0008 \\
\hline config. 5 & & \\
-MLE parameters & .50 & .008 \\
-EM parameters & .50 & .008
\end{tabular}

TABLE III: $\alpha$ and $\beta$ parameters for signal distribution estimated from experiment

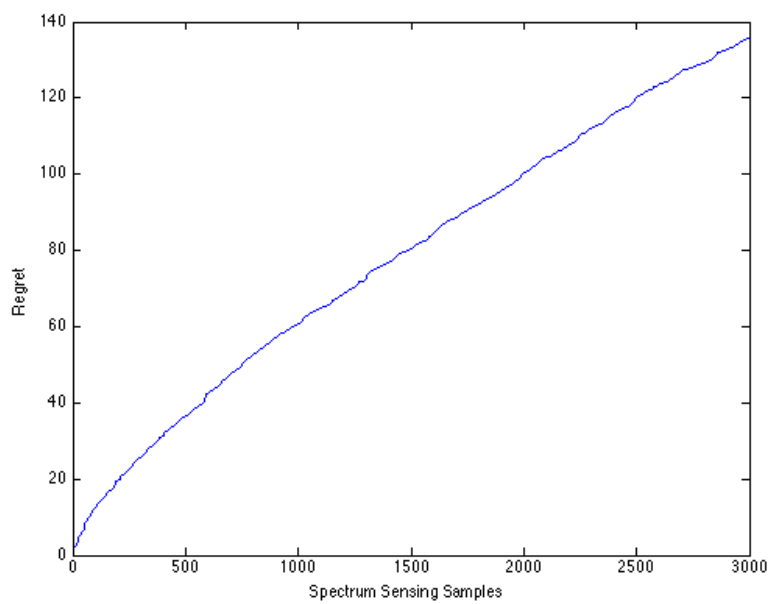

Fig. 8: Experimental Regret of the UCB policy.

ERAS estimates of the signal parameters are quite accurate despite low SNR. Results indicate that the UCB algorithm is capable of accurately estimating sensor performance. This result is notable particularly for antenna configurations that are rarely used. Reliable parameter estimates for rarely used antenna configurations can be obtained from minimal samples, making it possible to improve the effective performance from the frequent use of optimal antenna configurations.

\section{CONCLUSION}

An online algorithm is proposed for the optimal selection of ERAS antenna configurations for the purpose of spectrum sensing. This configuration selection algorithm minimizes the overhead required to estimate the performance of each antenna configuration, allowing optimal exploitation of the optimal antenna states for the desired objective. In addition, the use of the EM algorithm for Gamma mixtures enables the reliable estimation of spectrum sensing performance without directly observing whether spectrum sensing decisions are made correctly.

Experimental measurements taken in an indoor office environment validate the approach, and demonstrate that the
ERAS selection algorithm achieves rapid convergence in time and provides robust performance estimates even in low SNR scenarios. Measured results show that the ERAS can be leveraged to exploit pattern and polarization diversity to improve spectrum sensing performance. Computationally simple algorithms make it possible for DSA networks to improve CSI reliability locally with little overhead. Additionally, the approach provided is flexible enough to work with any type of reconfigurable antenna with minimal modification.

While results indicate that an omni-directional antenna pattern works well for energy detectors in office environments with rich multi-path scattering, the use of directional antenna patterns has been proven to provide gains in environments where multi-path is sparse. Additionally, spectrum sensors that rely on more complex analysis of the spectrum may benefit from the spatial filtering offered by directional patterns. In both cases, the added diversity of pattern and polarization can demonstrate improvements in both sensing and access performance through the use of intelligent antenna configuration control.

\section{ACKNOWLEDGEMENT}

The research described in this paper was performed as part of the WiFiUS (Wireless Innovation between Finland and US) program and was supported by the National Science Foundation under grant 1147838.

\section{REFERENCES}

[1] T. Yucek and H. Arslan, "A survey of spectrum sensing algorithms for cognitive radio applications," Communications Surveys Tutorials, IEEE, vol. 11, no. 1, pp. 116-130, 2009.

[2] B. Agee, S. Schell, and W. Gardner, "Spectral self-coherence restoral: a new approach to blind adaptive signal extraction using antenna arrays," Proceedings of the IEEE, vol. 78, no. 4, pp. 753-767, 1990.

[3] K. Du and W. Mow, "Affordable cyclostationarity-based spectrum sensing for cognitive radio with smart antennas," Vehicular Technology, IEEE Transactions on, vol. 59, no. 4, pp. 1877-1886, 2010.

[4] S. Yu and X. Wang, "Joint spectrum sensing in distributed MIMO systems," in Vehicular Technology Conference (VTC Spring), 2011 IEEE 73rd, 2011, pp. 1-4.

[5] R. Zhang, T. J. Lim, Y.-C. Liang, and Y. Zeng, "Multi-antenna based spectrum sensing for cognitive radios: A GLRT approach," Communications, IEEE Transactions on, vol. 58, no. 1, pp. 84-88, 2010.

[6] G. Zhong, J. Guo, Z. Zhao, and D. Qu, "Cyclostationarity based multiantenna spectrum sensing in cognitive radio networks," in Vehicular Technology Conference (VTC 2010-Spring), 2010 IEEE 71st, 2010, pp. $1-5$.

[7] A. Taherpour, M. Nasiri-Kenari, and S. Gazor, "Multiple antenna spectrum sensing in cognitive radios," Wireless Communications, IEEE Transactions on, vol. 9, no. 2, pp. 814-823, 2010.

[8] Y.-C. Liang, G. Pan, and Y. Zeng, "On the performance of spectrum sensing algorithms using multiple antennas," in Global Telecommunications Conference (GLOBECOM 2010), 2010 IEEE, 2010, pp. 1-5.

[9] J. T. Bernhard, "Reconfigurable antennas," Synthesis lectures on antennas, vol. 2, no. 1, pp. 1-66, 2007.

[10] D. Piazza, M. D’Amico, and K. Dandekar, "Two port reconfigurable CRLH leaky wave antenna with improved impedance matching and beam tuning," in Proceedings of the 2009 European Conference on Antennas Propagation (EuCAP), 2009.

[11] D. Patron, D. Piazza, and K. Dandekar, "A wideband planar antenna with omnidirectional and directional radiation patterns," in IEE Electronics Letters, 2013.

[12] A. Grau, J. Romeu, M.-J. Lee, S. Blanch, L. Jofre, and F. de Flaviis, "A dual-linearly-polarized MEMS-reconfigurable antenna for narrowband MIMO communication systems," Antennas and Propagation, IEEE Transactions on, vol. 58, no. 1, pp. 4-17, 2010. 
[13] A. Grau, H. Jafarkhani, and F. de Flaviis, "A reconfigurable multipleinput multiple-output communication system," Wireless Communications, IEEE Transactions on, vol. 7, no. 5, pp. 1719-1733, 2008.

[14] D. Piazza, J. Kountouriotis, M. D' Amico, and K. Dandekar, "A technique for antenna configuration selection for reconfigurable circular patch arrays," Wireless Communications, IEEE Transactions on, vol. 8, no. 3, pp. 1456-1467, 2009.

[15] N. Gulati and K. Dandekar, "Learning state selection for reconfigurable antennas: A multi-armed bandit approach," Antennas and Propagation, IEEE Transactions on, vol. 62, no. 3, pp. 1027-1038, March 2014.

[16] A. Abdi and M. Kaveh, "On the utility of gamma PDF in modeling shadow fading (slow fading)," in Vehicular Technology Conference, 1999 IEEE 49th, vol. 3, Jul 1999, pp. 2308-2312 vol.3.

[17] P. Shankar, "Error rates in generalized shadowed fading channels," Wireless Personal Communications, vol. 28, no. 3, pp. 233-238, 2004.

[18] A. Dempster, N. Laird, and D. Rubin, "Maximum likelihood from incomplete data via the EM algorithm," vol. 39, no. 1, pp. 1-38, 1977.

[19] C. J. Wu, "On the convergence properties of the EM algorithm," The
Annals of statistics, pp. 95-103, 1983

[20] S. M. Kay, Fundamentals of Statistical Signal Processing, Volume 2: Detection Theory. Prentice Hall PTR, Jan. 1998.

[21] P. Auer, N. Cesa-Bianchi, and P. Fischer, "Finite-time analysis of the multiarmed bandit problem," vol. 47, May 2002.

[22] D. Patron, D. Piazza, and K. Dandekar, "Wideband planar antenna with reconfigurable omnidirectional and directional radiation patterns," Electronics Letters, vol. 49, no. 8, 2013.

[23] R. University, "WARP project." [Online]. Available: http://warp.rice.edu

[24] A. M. Sayeed and V. Raghavan, "Maximizing mimo capacity in sparse multipath with reconfigurable antenna arrays," Selected Topics in Signal Processing, IEEE Journal of, vol. 1, no. 1, pp. 156-166, 2007.

[25] R. Bahl, N. Gulati, K. Dandekar, and D. Jaggard, "Impact of pattern reconfigurable antennas on interference alignment over measured channels," in Globecom Workshops (GC Wkshps), 2012 IEEE, Dec 2012, pp. 557-562. 